\title{
92 Temporal bone fractures
}

A About $80 \%$ are longitudinally placed.

B The sensorineural hearing loss associated with longitudinal fractures does not recover.

C Dysequilibrium may be caused by physical fatigue.

D Facial paralysis is seen in about $50 \%$ of transverse fractures.

E Cawthorne-Cooksey exercises may expedite vestibular compensation.

\section{In closed head injuries without fracture}

A Hearing loss, if present, is most profound at $4000 \mathrm{~Hz}$.

$B$ The most common vestibular symptom is positional vertigo.

C Unilateral canal paresis is common.

D Latent nystagmus may be revealed by removal of optic fixation.

E Rapid acceleration may cause cochlear vestibular damage.

\section{Inner ear barotrauma}

A A Valsalva manoeuvre, during a deep sea dive, in the presence of a locked eustachian tube may cause labyrinthine window rupture.

B Decompression sickness is best treated by giving an oxygenhelium breathing mixture.

C Patients with a short wide cochlear aqueduct are more prone to problems.

D A previous stapedectomy reduces the risk of damage during flying.

E Unilateral labyrinthine failure can be caused by changing from an oxygen-helium gas mix to compressed air at the start of decompression. 